\title{
Correction to: Mastering Uncertainty in Mechanical Engineering
}

Peter F. Pelz, Peter Groche, Marc E. Pfetsch, and Maximilian Schaeffner

\section{Correction to:}

P. Pelz et al. (eds.), Mastering Uncertainty in Mechanical Engineering, Springer Tracts in Mechanical Engineering, https://doi.org/10.1007/978-3-030-78354-9

In the original version of the book, the following corrections have been incorporated:

On page 49, the last equation in the text has been changed.

On page 95, '\#' has been replaced with $\vartheta$ in Eqs. 3.28 and 3.29.

In Chap. 5, the author name has been changed from Nasssr AlBaradoni to Nassr Al-Baradoni.

The book and the chapters have been updated with the changes.

The updated version of this chapters can be found at https://doi.org/10.1007/978-3-030-78354-9_3 and https://doi.org/10.1007/978-3-030-78354-9_5 